\title{
DS-CDMA Chip Waveform Design for Minimal Interference Under Bandwidth, Phase, and Envelope Constraints
}

\author{
Mohamed A. Landolsi and Wayne E. Stark, Fellow, IEEE
}

\begin{abstract}
This paper investigates the effect of chip waveform shaping on the error performance, bandwidth confinement, phase continuity, and envelope uniformity in direct-sequence codedivision multiple-access communication systems employing offset quadrature modulation formats. An optimal design methodology is developed for the problem of minimizing the multipleaccess interference power under various desirable signal constraints, including limited $99 \%$ and $99.9 \%$ power bandwidth occupancies, continuous signal phase, and near-constant envelope. The methodology is based on the use of prolate spheroidal wave functions to obtain a reduced-dimension discrete constrained optimization problem formulation. Numerous design examples are discussed to compare the performance achieved by the optimally-designed chip waveforms with other conventional schemes, such as offset quadrature phase-shift keying, minimumshift keying (MSK), sinusoidal frequency-shift keying (SFSK), and time-domain raised-cosine pulses. In general, it is found that while the optimized chip pulses achieved substantial gains when no envelope constraints were imposed, these gains vanish when a low envelope fluctuation constraint was introduced. In particular, it is also shown that MSK is quasi-optimal with regard to the 99\% bandwidth measure, while the raised-cosine pulse is equally good with both the $99 \%$ and $99.9 \%$ measures, but at the expense of some envelope variation. On the other hand, SFSK is quasioptimal with regard to the $99.9 \%$ bandwidth occupancy, among the class of constant-to-low envelope variation pulses.
\end{abstract}

Index Terms - Chip waveform optimization, direct-sequence CDMA, envelope uniformity, multiple-access interference, phase continuity.

\section{INTRODUCTION}

$\mathbf{I}$ $\mathrm{N}$ THIS paper, we consider the performance of directsequence code-division multiple-access (DS-CDMA) communication systems with signaling schemes that use offset linear quadrature modulation with arbitrary chip waveform shapes, random signature sequences, and single-user correlation receivers. Common examples of this category of signaling schemes are offset quadrature phase-shift keying (OQPSK) with the conventional rectangular chip waveform

Paper approved by U. Madhow, the Editor for Spread Spectrum of the IEEE Communications Society. Manuscript received August 15, 1997; revised September 15,1998 . This work was supported in part by the National Science Foundation under Grant NCR-9115969.

M. A. Landolsi is with Nortel Networks, Wireless Solutions Division, Ottawa, ON K1Y 4H7, Canada (e-mail: landolsi@nortelnetworks.com).

W. E. Stark is with the Department of Electrical Engineering and Computer Science, University of Michigan, Ann Arbor, MI 48109 USA (e-mail: stark@eecs.umich.edu).

Publisher Item Identifier S 0090-6778(99)08915-1. and minimum-shift keying (MSK) with the half-sine chip waveform [1], [2].

It is well known that appropriate data pulse shaping in linear quadrature modulation improves the spectral performance of the modulation scheme because smooth signal transitions yield a fast rolloff of the power spectrum of the signal and improve its spectral confinement. Such improved bandwidth efficiency is highly desirable in DS-CDMA applications because it allows for the use of longer spreading codes at a given allocated bandwidth, thereby improving the error performance of the system for a given number of users or making it possible to accommodate a larger number of users for a given bit-error rate. In addition, for DS-CDMA systems with a correlation receiver, pulse shaping by itself directly impacts error performance. This is because the variance of the multiple-access interference depends on the actual shape of the chip waveform and not only on its energy [1]. This is unlike communication over channels corrupted by additive white Gaussian noise only, where pulses with different shapes but with equal energy still have the same error performance.

There is comparatively little work done on the optimization of chip waveforms for DS-CDMA systems. Instead, more effort was devoted to the design of good spreading sequences in order to better reduce interference and mitigate multipath effects. This was also combined with the use of coding and diversity schemes to further improve performance. Previous results related to chip waveform design were presented in [3] where suboptimum solutions to the problem of designing pulses that achieve minimum squared correlation for a given inband power were constructed based on the use of prolate spheroidal wave functions [16]. For strictly bandlimited pulses (which therefore have infinite time duration), this problem was discussed in [18] and the optimal pulses are found to have a constant spectrum, i.e., with a $\sin (x) / x$ shape in time-domain, as was also shown earlier in [3]. Chip waveform optimization was similarly considered with binary phase-shift keying (BPSK) modulation in [10], where direct calculus of variation techniques were used to minimize the multiple-access interference variance, but without considering the additional figures of merit that we incorporate in this work (namely, the bandwidth, phase, and envelope constraints, as will be discussed shortly). Another problem involving pulse shape design was considered in [11], where minimum rms bandwidth is considered for a set of direct-sequence signals with a specified correlation matrix. Also, optimal pulse shaping 
was considered in [12] for a different system model based on orthogonal multicarrier quasi-synchronous CDMA. Additional results that highlight the differences in performance (mainly bit-error probability) between several known pulses can be found in [8] and [13], and the importance of chip waveform shaping in the mitigation of multipath effects in DS-CDMA was also addressed in [14].

In this paper, following the work in [4] and [5], we consider the case of full-response chip pulses, i.e., those limited to a single chip interval and extend the design requirements to solve for the optimal chip waveforms that achieve minimal multiple-access interference variance while preserving several important signal features, including fixed total signal power, limited inband power bandwidth, phase continuity, and envelope uniformity, which are necessary to preserve the spectral performance of the signaling schemes, especially when power-efficient nonlinear amplifiers are used. The results obtained provide extensive performance comparisons and benchmarking for several families of optimal chip waveforms designed with various constraints and at different bandwidth occupancy levels. In addition, the potential achievable gains in performance of these optimal waveforms over some of the conventional modulation schemes (e.g., OQPSK and other MSK-type modulations) are also quantified. We also point out that although our focus in this paper is limited to the family of full-response pulse shapes, the optimization methodology used is still applicable to the general case of partial-response pulses with only a few modifications to account for the different signal format.

The rest of the paper is organized as follows. In Section II, the system and signal models used are discussed. In Section III, we formulate the optimization problem and define the various constraints. In Section IV, the solution methodology is discussed based on the use of prolate spheroidal wave functions. Several design examples and numerical results are given in Section V, and final conclusions are presented in Section VI.

\section{SySTEM MODEL}

We use the classical framework and notation of [1] and [2], which focuses on the many-to-one system topology that models the reverse (mobile-to-base) link of a single-cell DSCDMA system. We briefly review this model here. Assuming $K$ active users with transmitted signals of the generalized OQPSK format, the information bits of user $k, 1 \leq k \leq K$, are split into inphase and quadrature streams

$$
\begin{aligned}
& b_{k}^{I}(t)=\sum_{l=-\infty}^{\infty} b_{k, l}^{I} p_{T}(t-l T) \\
& b_{k}^{Q}(t)=\sum_{l=-\infty}^{\infty} b_{k, l}^{Q} p_{T}(t-l T)
\end{aligned}
$$

where $p_{T}(t)$ is the unit pulse on the bit interval $[0, T]$, and the data bits $b_{k l}^{I}$ and $b_{k l}^{Q}$ are binary random variables $\in\{+1,-1\}$ with equal probability. The data streams are multiplied by the spectrum-spreading signals

$$
\begin{aligned}
& a_{k}^{I}(t)=\sum_{m=-\infty}^{\infty} a_{k, m}^{I} \psi_{c}\left(t-m T_{c}\right) \\
& a_{k}^{Q}(t)=\sum_{m=-\infty}^{\infty} a_{k, m}^{Q} \psi_{c}\left(t-m T_{c}\right)
\end{aligned}
$$

where $\left(a_{k}^{I}\right)$ and $\left(a_{k}^{Q}\right)$ are two distinct signature sequences modeled as random, aperiodic, with independent, equally likely chip symbols $a_{k, m}^{I}, a_{k, m}^{Q} \in\{+1,-1\}$. We assume that each bit is coded with $N$ chips (i.e., $T=N T_{c}$ ). The chip waveform $\psi_{c}(t)$ is time-limited to the interval $\left[0, T_{c}\right]$ and normalized to have energy $T_{c}$, so that

$$
\int_{0}^{T_{c}} \psi_{c}^{2}(t) d t=T_{c}
$$

The two spread signals are modulated onto two carriers in quadrature, with a time offset of half a chip period $T_{c} / 2$ introduced in the cosine branch. The resulting inphase and quadrature signals are

$$
\begin{aligned}
& s_{k}^{I}(t)=a_{k}^{I}\left(t-\frac{T_{c}}{2}\right) b_{k}^{I}\left(t-\frac{T_{c}}{2}\right) \cos \left(\omega_{c} t+\theta_{k}\right) \\
& s_{k}^{Q}(t)=a_{k}^{Q}(t) b_{k}^{Q}(t) \sin \left(\omega_{c} t+\theta_{k}\right)
\end{aligned}
$$

where $\theta_{k}$ is the carrier phase angle and $\omega_{c}$ its angular frequency. The resulting transmitted signal from the $k$ th user is obtained by the sum

$$
s_{k}(t)=s_{k}^{I}(t)+s_{k}^{Q}(t) .
$$

At the front end of the receiver, the signal present $r(t)$ is the sum of delayed versions of the transmitted signals of all active users, with additive thermal noise

$$
\begin{aligned}
r(t)= & n(t)+\sqrt{2 P} \sum_{k=1}^{K} a_{k}^{I}\left(t-\tau_{k}\right) b_{k}^{I}\left(t-\tau_{k}\right) \sin \left(\omega_{c} t+\phi_{k}\right) \\
& +\sqrt{2 P} \sum_{k=1}^{K} a_{k}^{Q}\left(t-\frac{T_{c}}{2}-\tau_{k}\right) b_{k}^{Q}\left(t-\frac{T_{c}}{2}-\tau_{k}\right) \\
& \cdot \cos \left(\omega_{c} t+\phi_{k}\right)
\end{aligned}
$$

where $n(t)$ is a white Gaussian process with power spectral density (PSD) $N_{0} / 2$ modeling the thermal noise, $P$ is the received signal power in each inphase and quadrature component, assumed to be the same for all users. This "perfect power control" assumption is only used for simplicity and has no real impact on the validity of the subsequent results and conclusions. $\tau_{k}$ is the $k$ th signal time delay, and $\phi_{k}$ its cumulated phase equal to $\theta_{k}-\omega_{c} \tau_{k}$. The system is assumed to be asynchronous, so different users have independent time delays and phases, where delays are interpreted modulo- $T$ and modeled as uniform random variables on the interval $[0, T]$, and phases are interpreted modulo- $2 \pi$ and modeled as uniform random variables on $[0,2 \pi]$. We consider single-user coherent correlation receivers in this paper. Because of the symmetry of the model, we only focus on the quadrature signal of user one, and since perfect synchronization is assumed, we also set $\tau_{1}=\phi_{1}=0$. All other delays and phases are then interpreted relative to user one. The desired receiver decision statistic is then given by

$$
Z_{1}^{Q}(t)=\int_{t-T}^{t} r(\tau) a_{1}^{Q}(\tau) \sin \left(\omega_{c} \tau\right) d \tau
$$

The decision statistic is sampled at multiples of the bit duration $T$, followed by a zero-threshold decision device that outputs a symbol $\hat{b}_{1, i-1}^{Q}=1$ if $Z_{1}^{Q}(i T)>0$ and a symbol $\hat{b}_{1, i-1}^{Q}=-1$ otherwise. 


\section{Choice OF ChIP WAVEForm}

The model presented in the previous section is valid for arbitrary chip waveforms $\psi_{c}(t)$. We now discuss different criteria for the selection of a good waveform shape for $\psi_{c}(t)$. We focus on the criteria that pertain to the spectral occupancy of the transmitted signal and their phase continuity and envelope uniformity. The effect of the chip waveform on the error performance, which is very important, is also discussed. For simplicity, we will limit attention to chip waveforms that are continuous on $\left[0, T_{c}\right]$, with even symmetry about $T_{c} / 2$.

\section{A. Bandwidth Performance}

The bandwidth performance of the signaling schemes we are considering is the same as any linear modulation format (binary or quaternary, spread or unspread) with the same data (or chip) pulse shape and duration. This performance is characterized by the PSD of the baseband signals. Since in our model the data and chip symbols are independently, identically distributed binary random variables from $\{+1,-1\}$, it follows that the PSD is continuous (i.e., has no line components) and is proportional to the squared magnitude of the Fourier transform of the chip waveform. This PSD is given by

$$
S_{\psi_{c}}(f)=\frac{4 P}{T_{c}}\left|\int_{0}^{T_{c}} \psi_{c}(t) e^{-j 2 \pi f t} d t\right|^{2} .
$$

A common figure of merit for the evaluation of bandwidth performance is the compactness of the main lobe of the PSD, expressed as the null-to-null bandwidth. This depends on the duration and shape of the chip waveform and can be reduced if longer chip pulses with duration exceeding $T_{c}$ are used. This is a case of partial-response signaling, and we only consider full-response signals with pulses limited to $\left[0, T_{c}\right]$ in this paper.

Another criterion that characterizes bandwidth performance is the rate of decay, or rolloff, of the power spectrum. Faster decay is obtained by using smoother pulses, where smoothness is translated by having time derivatives of the waveform vanishing to zero at the endpoints of the interval $\left[0, T_{c}\right]$. For linear modulation schemes, it is known (see, for example, [20]) that if the data pulse has all its derivatives up to the $\mu$ th order equal to zero at the endpoints of the data interval, then its PSD decays as $|f|^{-(4 \mu+2)}$. It should be mentioned, however, that this decay rate is only asymptotic and typically occurs far away from the main lobe. Hence, it may be irrelevant for practical purposes. We note here that imposing the above condition on $\psi_{c}(t)$ only (i.e., with $\mu=0$ ) guarantees the phase continuity of modulated signals, in which case, the PSD decays at least as $|f|^{-2}$. This is further illustrated in the next section.

Another commonly used bandwidth measure is the inband power bandwidth [20]. This is defined as the frequency band that contains a given fraction $1-\eta$ of the signal power, where $\eta$ represents the fraction of out-of-band power and is such that $0 \leq \eta \leq 1$. If we let $W$ denote the one-sided bandwidth occupancy, then we have

$$
\frac{\int_{-W}^{W} S_{\psi_{c}}(f) d f}{\int_{-\infty}^{\infty} S_{\psi_{c}}(f) d f}=1-\eta
$$

Commonly used values for $\eta$ are 0.01 and 0.001 , corre- sponding to the $99 \%$ and $99.9 \%$ bandwidth occupancies, respectively. In the rest of the paper, we mainly use these two figures as a measure for comparing the bandwidth performance of different chip waveforms.

\section{B. Envelope Uniformity}

From the signal expressions in (6) and (7), it is seen that the signal envelope is given by

$$
e(t)= \begin{cases}\sqrt{\psi_{c}^{2}(t)+\psi_{c}^{2}\left(t+\frac{1}{2} T_{c}\right)}, & 0 \leq t<\frac{1}{2} T_{c} \\ \sqrt{\psi_{c}^{2}(t)+\psi_{c}^{2}\left(t-\frac{1}{2} T_{c}\right)}, & \frac{1}{2} T_{c} \leq t<T_{c} .\end{cases}
$$

Identical expressions hold for $t \in\left[i T_{c},(i+1) T_{c}\right]$ with $i>0$ because of the periodicity of $e(t)$. Notice also that the two expressions in (13) are identical because of the even symmetry of $\psi_{c}(t)$ about $T_{c} / 2$. Ideally, $e(t)$ should be constant for all $t$. If this is not the case, the amount of envelope variation, which is quantified by the amplitude modulation (AM) index

$$
\Delta e=\frac{\max (e(t))-\min (e(t))}{\max (e(t))+\min (e(t))}
$$

should be kept at a low level. Such a requirement on envelope uniformity is especially important in the presence of nonlinear processing circuits such as power-efficient nonlinear amplifiers. More precisely, it is known that if the carrier signal envelope is not constant, spectral regrowth (i.e., regeneration of sidelobes in the power spectrum) will occur at the final nonlinear amplifier stage of the transmitter [15]. Hence, it is important to design modulation formats with a constant (or near-constant) envelope.

For binary modulation schemes, unless the chip pulse is itself constant over $\left[0, T_{c}\right]$ (such as in standard BPSK), the carrier envelope will be rapidly changing following the time fluctuations of the chip pulse. For offset quadrature modulation, however, it is possible to use nonconstant smoothly varying chip pulses (for bandwidth reduction purposes) and still obtain a constant envelope or at least maintain a low AM index. This will be further illustrated with the subsequent chip waveform examples.

\section{Phase Continuity}

It is also desirable to use continuous-phase signals because their smooth phase transitions yield a more compact power spectrum with rapidly decaying sidelobes and are less susceptible to transient and high-order harmonic generation by hardware circuitry [20]. In addition, phase continuity is also important in relation to envelope uniformity and nonlinear amplification. Indeed, it is known [6] that if a signal with phase discontinuities is run through a channel band-limiting filter, its envelope undergoes time fluctuations with a magnitude increasing with the amount of phase jump incurred. Since channel filtering is always present ${ }^{1}$ and is usually followed by nonlinear power-efficient amplification, it is highly desirable to impose phase continuity on the transmitted signals, so as to preserve their spectral confinement.

\footnotetext{
${ }^{1}$ Although not included in this paper, the effects of filtering are important and are being deferred to later work, for space considerations.
} 
As was mentioned previously, phase continuity is obtained by having the pulse $\psi_{c}(t)$ go to zero at the chip interval endpoints, i.e., $\psi_{c}(0)=\psi_{c}\left(T_{c}\right)=0$. To see this, we rewrite, as in [9], the $k$ th transmitted signal in the following form

$$
s_{k}(t)=\sqrt{2 P} e(t) \cos \left(\omega_{c} t-\phi_{a}(t)+\theta_{k}\right)
$$

where additional phase term $\phi_{a}(t)$ is given by

$$
\phi_{a}(t)=\left\{\begin{array}{c}
-\tan ^{-1}\left[\frac{b_{l}^{Q} a_{m}^{Q} \psi_{c}\left(t-(l N+m) T_{c}\right)}{b_{l}^{I} a_{m-1}^{I} \psi_{c}\left(t-\left(l N+m-\frac{1}{2}\right) T_{c}\right)}\right], \\
(l N+m) T_{c} \leq t<\left(l N+m+\frac{1}{2}\right) T_{c} \\
-\tan ^{-1}\left[\frac{b_{l}^{Q} a_{m}^{Q} \psi_{c}\left(t-(l N+m) T_{c}\right)}{b_{l}^{I} a_{m}^{I} \psi_{c}\left(t-\left(l N+m+\frac{1}{2}\right) T_{c}\right)}\right], \\
\left(l N+m+\frac{1}{2}\right) T_{c} \leq t<(l N+m+1) T_{c}
\end{array}\right.
$$

Recall that the products $b_{l}^{Q} a_{m}^{Q}$ and $b_{l}^{I} a_{m}^{I}$ can only change value at time instants equal to a multiple of $T_{c}$ or $T_{c} / 2$ plus a multiple of $T_{c}$, respectively. Therefore, assuming the pulse $\psi_{c}(t)$ to be time-continuous inside the chip interval, it is clear that the phase $\phi_{a}(t)$ will have no jumps if we constrain $\psi_{c}(t)$ to be zero at the instants when the terms $b_{l}^{Q} a_{m}^{Q}$ and $b_{l}^{I} a_{m}^{I}$ may change. Hence, the condition for phase continuity is obtained by forcing the chip waveform to zero at the limits of the chip interval

$$
\psi_{c}(0)=\psi_{c}\left(T_{c}\right)=0 .
$$

Because of the time symmetry of $\psi_{c}$ about $T_{c} / 2$, only one equality is sufficient. If this condition is not met, it is seen that a phase jump of absolute magnitude

$$
\Delta \phi=\left|\tan ^{-1}\left[\frac{\psi_{c}\left(\frac{T_{c}}{2}\right)}{\psi_{c}\left(0^{+}\right)}\right]+\tan ^{-1}\left[\frac{\psi_{c}\left(\frac{T_{c}}{2}\right)}{\psi_{c}\left(T_{c}^{-}\right)}\right]\right|
$$

can occur in the middle of the interval considered, at $t=$ $\left(l N+m+(1 / 2) T_{c}\right)$. The + and - superscripts indicate limits as $t$ approaches 0 and $T_{c}$ from the right and the left, respectively. Again, by symmetry of $\psi_{c}(t), \Delta \phi$ simplifies to

$$
\Delta \phi=2\left|\tan ^{-1}\left[\frac{\psi_{c}\left(\frac{T_{c}}{2}\right)}{\psi_{c}\left(0^{+}\right)}\right]\right| \bmod (\pi) .
$$

Notice that because $\Delta \phi$ is interpreted modulo- $\pi$, the above expression is also equivalent to

$$
\Delta \phi=2\left|\tan ^{-1}\left[\frac{\psi_{c}\left(0^{+}\right)}{\psi_{c}\left(\frac{T_{c}}{2}\right)}\right]\right| \bmod (\pi)
$$

which follows from the identity $\tan ^{-1}(x)+\tan ^{-1}(1 / x)=$ $\pi / 2$.

\section{Bit-Error Performance}

It is well known from previous pioneering work on DSCDMA [1] that the shape of the chip waveform also affects bit-error performance. The exact evaluation of the bit-error probability $P_{b}$ is complex and requires the full characterization of the statistics of the multiple-access interference terms. In our case, for the purpose of comparing different chip waveforms, it is sufficient to consider the average bit-energy-to-noiseplus-interference density ratio, which, for simplicity, will be referred to as the signal-to-noise ratio (SNR) defined by the ratio of the squared expected value of the decision statistic to its variance. The bit-error probability is then approximated by $P_{b} \simeq Q[\sqrt{\mathrm{SNR}}]$, where $Q$ denotes the error function $Q(x)=$ $(1 / \sqrt{2 \pi}) \int_{x}^{\infty} e^{-u^{2} / 2} d u$. The SNR expression is mathematically more tractable and yields a good approximation to the average bit-error probability in the case of a large number of users, which is of practical interest. For the case of offset quadrature schemes with random spreading sequences that we are considering here, it was readily shown [1]-[4] that the SNR expression can be obtained as

$$
\mathrm{SNR}=\frac{\left[E\left[Z_{1}^{Q}(T)\right]\right]^{2}}{\operatorname{var}\left[Z_{1}^{Q}(T)\right]}=\frac{2 E_{b}}{N_{0}+4(K-1) E_{b} \frac{M_{c}}{N}}
$$

where $E_{b}$ denotes the energy per bit $P T$, and $M_{c}$ is the normalized mean-squared partial chip correlation

$$
M_{c}=\frac{1}{T_{c}^{3}} \int_{0}^{T_{c}} R_{\psi_{c}}^{2}(s) d s=\frac{1}{T_{c}^{3}} \int_{0}^{T_{c}} \hat{R}_{\psi_{c}}^{2}(s) d s
$$

which is obtained from the continuous-time partial autocorrelation functions [1] given by $R_{\psi_{c}}(s)=\int_{0}^{s} \psi_{c}(t) \psi_{c}\left(t+T_{c}-s\right) d t$ and $\hat{R}_{\psi_{c}}(s)=\int_{s}^{T_{c}} \psi_{c}(t) \psi_{c}(t-s) d t$.

The SNR clearly depends on the number chips per bit (or processing gain) $N$, but also for our purpose, on the actual chip waveform shape itself through the correlation parameter $M_{c}$. The smaller the ratio $M_{c} / N$, the higher the SNR and the better the error performance. However, in order to fairly compare modulations using different chip waveforms, we must impose equal bit rate and equal bandwidth constraints. This requires that $N$ and $T_{c}$ be modified with each chip waveform. For example, if we consider two systems using two different modulations and let $B^{(1)}$ and $B^{(2)}$ denote their respective normalized bandwidth occupancies, ${ }^{2}$ then we have to maintain

$$
N^{(1)} B^{(1)}=N^{(2)} B^{(2)}
$$

and clearly, if $B^{(1)}$ is larger than $B^{(2)}$, System 2 will have a larger processing gain. To determine which system will have a better error performance, we must also look at the ratio of the normalized chip squared correlation factors. Consider for simplicity the SNR expression without the thermal noise term (i.e., $N_{0}=0$ ), then the gain (or loss) in SNR of System 2 over System 1 is given by

$$
G_{\mathrm{SNR}}=10 \log _{10}\left[\frac{B^{(1)} M_{c}^{(1)}}{B^{(2)} M_{c}^{(2)}}\right] \mathrm{dB}
$$

which clearly demonstrates the importance of the normalizedbandwidth mean-squared correlation product $B M_{c}$ as a single

\footnotetext{
${ }^{2}$ The normalization is by the respective chip durations $T_{c}^{(1)}$ and $T_{c}^{(2)}$, i.e., $B^{(1)}$ and $B^{(2)}$ are dimensionless numbers given by $W^{(1)} T_{C}^{(1)}$ and $W^{(2)} T_{C}^{(2)}$, respectively.
} 
TABLE I

Comparison of Modulations Employing Different Chip WaVeforms

\begin{tabular}{c|c|c|c|c|c|c}
\hline $\begin{array}{c}\text { Modulation } \\
\text { Chip Waveform }\end{array}$ & $\begin{array}{c}\text { Max } \\
\Delta \phi\end{array}$ & $\begin{array}{c}\text { AM } \\
\text { Index }\end{array}$ & $\begin{array}{c}\text { PSD } \\
\text { Decay }\end{array}$ & $M_{c}$ & $\begin{array}{c}9 \% \mathrm{Bw} \\
W T_{c}\end{array}$ & $\begin{array}{c}99.9 \% \mathrm{Bw} \\
W T_{c}\end{array}$ \\
\hline$\psi_{c}^{\text {OQPSK }}(l)=p_{T_{c}}(t)$ & $90^{\circ}$ & $0 \%$ & $|f|^{-2}$ & 0.3333 & 10.42 & 29.32 \\
\hline$\psi_{c}^{\mathrm{MSK}}(t)=\sqrt{2} \sin \left(\frac{\pi t}{T_{c}^{\prime}}\right) p_{T_{c}}(t)$ & $0^{\circ}$ & $0 \%$ & $|f|^{-4}$ & 0.2933 & 1.18 & 2.79 \\
\hline$\psi_{c}^{\mathrm{SFSK}}(t)=\sqrt{2 \sin \left(\frac{\pi t}{T_{c}}-\frac{1}{4} \sin \left(\frac{4 \pi t}{T_{c}}\right)\right) p_{T_{r}}(t)}$ & $0^{\circ}$ & $0 \%$ & $|f|^{-8}$ & 0.2457 & 2.20 & 2.87 \\
\hline$\psi_{c}^{\text {TDRC }}(t)=\sqrt{2 / 3}\left(1-\cos \left(\frac{2 \pi t}{T_{c}}\right)\right) p_{T_{c}}(t)$ & $0^{\circ}$ & $17 \%$ & $|f|^{-6}$ & 0.2405 & 1.40 & 1.72 \\
\hline
\end{tabular}

parameter for comparing the error performance of different chip pulses in DS-CDMA. Note also that the SNR differences can be traded for a corresponding variation in capacity (as measured by the number $K$ of simultaneous users) if the SNR is fixed for both systems. At equal SNR, the capacity gain (or loss) of System 2 over System 1 given by the percentage ${ }^{3}$

$$
G_{\mathrm{CAP}}=\left[\frac{B^{(1)} M_{c}^{(1)}}{B^{(2)} M_{c}^{(2)}}\right] \times 100 \% .
$$

To illustrate the above figures of merit, we use four examples of known modulations schemes, namely OQPSK, MSK, SFSK (as proposed in [7]), and time-domain raised-cosine shaped OQPSK [8], referred to as TDRC. ${ }^{4}$ The first three modulations are examples of constant-envelope modulations whereas the last one is a varying envelope scheme as can be seen from Table I.

\section{Optimization Problem Formulation}

\section{A. Objective Function}

It is clear from the discussion and results of Section III that when all other parameters are held constant, minimizing the mean-squared correlation functional $M_{c}$ (which depends on the chip waveform shape) maximizes the SNR and consequently minimizes the bit-error probability. Recall also that for a fixed chip duration $T_{c}$ and inband power bandwidth $W$, the waveform that achieves minimum $M_{c}$ yields a minimum normalized-bandwidth mean-squared correlation product and, hence, the best relative performance among all chip waveforms having normalized bandwidth $B=W T_{c}$. Our objective is then to design chip waveforms that minimize the functional $M_{c}$ under a number constraints that will be specified in the following section.

As was mentioned previously, we limit the minimization space to the set of chip waveforms with even symmetry about the midpoint $T_{c} / 2$. The case of arbitrary nonsymmetric chip waveforms remains to be further explored. Also, for reasons that will be clear shortly, it is easier to work in frequencydomain as in [3] and [5]. Letting $\hat{\mathcal{R}}_{\psi_{c}}(f)$ denote the Fourier transform of $\hat{R}_{\psi_{c}}(s)$ and using Parseval's theorem, we have from $(22)$

$$
M_{c}=\frac{1}{T_{c}^{3}} \int_{-\infty}^{\infty}\left|\hat{\mathcal{R}}_{\psi_{c}}(f)\right|^{2} d f
$$

\footnotetext{
${ }^{3}$ This follows from the approximation $K-1 \approx K$ for $K$ large and the fact that $M_{c} / N$ is proportional to $B M_{c}$.

${ }^{4}$ As opposed to the well-known frequency-domain raised-cosine pulse shaping, which is a case of partial-response signaling and is not considered in this paper.
}

Since the chip pulse $\psi_{c}(t)$ is zero outside $\left[0, T_{c}\right]$, it follows from the expression of $\hat{R}_{\psi_{c}}(s)$ that it is equal to the convolution of $\psi_{c}(s)$ with the time-reversed pulse $\tilde{\psi}_{c}(s) \triangleq \psi_{c}(-s)$, so that

$$
\begin{aligned}
\hat{\mathcal{R}}_{\psi_{c}}(f) & =\mathcal{F}\left[\int_{-\infty}^{\infty} \psi_{c}(t) \tilde{\psi}_{c}(s-t) d t\right]=\mathcal{F}\left[\psi_{c}(s) * \tilde{\psi}_{c}(s)\right] \\
& =\left|\Psi_{c}(f)\right|^{2}
\end{aligned}
$$

where $\mathcal{F}[\cdot]$ denotes the Fourier transform operator and $\Psi_{c}(f)$ is the Fourier transform of the chip pulse $\psi_{c}(s)$. Equation (26) then becomes

$$
M_{c}=\frac{1}{T_{c}^{3}} \int_{-\infty}^{\infty}\left|\Psi_{c}(f)\right|^{4} d f .
$$

For further simplification, we introduce the shifted version $\phi_{c}(t)$ of $\psi_{c}(t)$ defined by $\phi_{c}(t) \triangleq \psi_{c}\left(t+\left(T_{c} / 2\right)\right)$, which is even over $\left[-\left(T_{c} / 2\right),\left(T_{c} / 2\right)\right]$, and so its Fourier transform $\Phi_{c}(f)$ is also real and even. Then, using the fact that $\Phi_{c}(f)=$ $\Psi_{c}(f) e^{j \pi T_{c}}$, we rewrite (28) as

$$
M_{c}=\frac{1}{T_{c}^{3}} \int_{-\infty}^{\infty} \Phi_{c}^{4}(f) d f
$$

which is the final form of the objective functional to be minimized.

\section{B. Constraints}

The minimization of $M_{c}$ is to be carried under a number of constraints that incorporate desirable properties into the transmitted signals as discussed in Section III. These constraints are listed below as the following.

1) First, the normalized energy constraint expressed in (5) by $\int_{0}^{T_{c}} \psi_{c}^{2}(t) d t=T_{c}$ must be satisfied. In frequencydomain, this is equivalent to

$$
\int_{-\infty}^{\infty}\left|\Psi_{c}(f)\right|^{2} d f=\int_{-\infty}^{\infty} \Phi_{c}^{2}(f) d f=T_{c} .
$$

2) Second, since $\psi_{c}(t)$ has finite duration, it cannot be strictly band-limited. Instead, a limit on the amount of power outside a given frequency band $[-W, W]$ can be imposed. This is expressed as

$$
\int_{-W}^{W}\left|\Psi_{c}(f)\right|^{2} d f=\int_{-W}^{W} \Phi_{c}^{2}(f) d f=(1-\eta) T_{c}
$$

where $\eta$ is the fraction of out-of-band power, typically set to 0.01 or 0.001 .

3) As a third constraint, continuous-phase transitions are imposed on the transmitted signals. As shown in Section III, this is expressed by

$$
\psi_{c}(0)=\psi_{c}\left(T_{c}\right)=\phi_{c}\left( \pm T_{c} / 2\right)=0
$$

or equivalently

$$
\int_{-\infty}^{\infty} \Phi_{c}(f) \cos \left(\pi f T_{c}\right)=0 .
$$


4) A final constraint is related to the amount of envelope fluctuations of the transmitted signals. The strict constant envelope constraint of Section III and expressed by

$$
\psi_{c}^{2}(t)+\psi_{c}^{2}\left(t \pm T_{c} / 2\right)=2
$$

is difficult to handle in frequency-domain. Instead, we use a relaxed condition proposed in [9], which is found to yield a near-constant envelope by simply maintaining equal envelope values at $0, T_{c} / 4,3 T_{c} / 4$, and $T_{c}$. Given the even symmetry and the continuous-phase condition, this amounts to imposing $\psi_{c}\left(T_{c} / 2\right)=\sqrt{2} \psi_{c}\left(T_{c} / 4\right)$, or equivalently, $\phi_{c}(0)=\sqrt{2} \phi_{c}\left(T_{c} / 4\right)$. In frequencydomain, this is written as

$$
\int_{-\infty}^{\infty} \Phi_{c}(f)\left(1-\sqrt{2} \cos \left(\frac{1}{2} \pi f T_{c}\right)\right) d f=0 .
$$

\section{APPROACH to SOLUTION}

The optimization problem discussed above is summarized as

$$
\begin{array}{ll}
\text { Minimize } & \frac{1}{T_{c}^{3}} \int_{-\infty}^{\infty} \Phi_{c}^{4}(f) d f \\
\text { Subject to }\left\{\begin{array}{l}
\int_{-\infty}^{\infty} \Phi_{c}^{2}(f) d f=T_{c} \\
\int_{-W}^{W} \Phi_{c}^{2}(f) d f=(1-\eta) T_{c} \\
\int_{-\infty}^{\infty} \Phi_{c}(f) \cos \left(\pi f T_{c}\right) d f=0 \\
\int_{-\infty}^{\infty} \Phi_{c}(f)\left(1-\sqrt{2} \cos \left(\frac{1}{2} \pi f T_{c}\right)\right) d f=0 .
\end{array}\right.
\end{array}
$$

Given the complexity of the objective and constraint functionals, it is difficult to solve directly for $\Phi_{c}(f)$ using calculus of variation techniques. Instead, our approach is to replace the problem by an equivalent discrete formulation obtained from the projection of $\Phi_{c}(f)$ onto a suitable family of basis functions which form a complete set over the space of functions of interest. The choice of a proper set of basis functions is of paramount importance in the reduction of the dimensionality of the equivalent discrete optimization problem. In this regard, prolate spheroidal wave functions [16], [17], [19] are particularly well suited to the problem at hand. We discuss these functions and their properties next.

\section{A. Prolate Spheroidal Wave Functions}

In [16] and [17], the authors used prolate spheroidal wave functions in a number of interesting applications pertaining to time- and band-limited pulses of finite energy. In particular, general problems using arbitrary limited functions in one domain (time or frequency) that achieve highest energy concentration in the other domain are solved. We draw on the results and properties demonstrated in [16] and [17] for these basis functions, which make them particularly well suited to solve the optimization problem we are interested in. ${ }^{5}$

Consider the space $\mathcal{L}_{\infty}^{2}$ of all functions $F(f)$ with finiteenergy, i.e., satisfying $\int_{-\infty}^{\infty}|F(f)|^{2} d f<\infty$. Similarly, $\mathcal{L}_{W}^{2}$

\footnotetext{
${ }^{5}$ We actually use the formulation of [16] and [17] with an interchange of the variables $t$ and $-f$ to obtain the dual results applicable to our situation, also pointed out in [3].
}

TABLE II

Eigenvalues of Prolate Spheroidal Wave Functions for Some Values of $c$

\begin{tabular}{c|c|c|c|c|c|c}
\hline $\begin{array}{c}\text { Parameter } \\
c=\pi W T_{\mathrm{c}}\end{array}$ & \multicolumn{6}{c}{ Eigerivalues } \\
\cline { 2 - 7 } & $\lambda_{0}$ & $\lambda_{2}$ & $\lambda_{4}$ & $\lambda_{6}$ & $\lambda_{8}$ & $\lambda_{10}$ \\
\hline$\pi$ & $0.9810 \mathrm{E} 0$ & $0.2436 \mathrm{E} 0$ & $1.0660 \mathrm{E}-3$ & $4.8030 \mathrm{E}-7$ & $5.9719 \mathrm{E}-11$ & $2.8431 \mathrm{E}-15$ \\
\hline $2 \pi$ & $0.9999 \mathrm{E} 0$ & $0.9671 \mathrm{E} 0$ & $0.2747 \mathrm{E} 0$ & $3.4782 \mathrm{E}-3$ & $7.4656 \mathrm{E}-6$ & $5.8204 \mathrm{E}-9$ \\
\hline $3 \pi$ & $0.9999 \mathrm{E} 0$ & $0.9975 \mathrm{E} 0$ & $0.9176 \mathrm{E} 0$ & $0.3339 \mathrm{E} 0$ & $5.9345 \mathrm{E}-3$ & $2.6294 \mathrm{E}-5$ \\
\hline
\end{tabular}

denotes the space of functions satisfying $\int_{-W}^{W}|F(f)|^{2} d f<$ $\infty$. We also denote by $\mathcal{D}$ the subspace of $\mathcal{L}_{\infty}^{2}$ of all functions $F(f)$ with an inverse Fourier transform that vanishes for $t \notin\left[-T_{c} / 2, T_{c} / 2\right]$. Then, for a given frequency $W$ and a time duration $T_{c}$, there exists a countably infinite set of real functions $\left\{\Phi_{i}(f)\right\}_{i=0}^{\infty}$ called prolate spheroidal wave functions and a set of associated real positive eigenvalues $\left\{\lambda_{i}\right\}_{i=0}^{\infty}$ with the following properties [16].

1) $\left\{\Phi_{i}(f)\right\}_{i=0}^{\infty}$ are complete and orthonormal in $\mathcal{D}$

$$
\int_{-\infty}^{\infty} \Phi_{i}(f) \Phi_{j}(f) d f= \begin{cases}1, & i=j \\ 0, & i \neq j\end{cases}
$$

2) $\left\{\Phi_{i}(f)\right\}_{i=0}^{\infty}$ are also complete and orthogonal in $\mathcal{L}_{W}^{2}$

$$
\int_{-W}^{W} \Phi_{i}(f) \Phi_{j}(f) d f= \begin{cases}\lambda_{i}, & i=j \\ 0, & i \neq j .\end{cases}
$$

It is also shown that $\Phi_{i}(f)$ is even for $i$ even and odd for $i$ odd. Both the wave functions $\left\{\Phi_{i}(f)\right\}_{i=0}^{\infty}$ and the associated eigenvalues $\left\{\lambda_{i}\right\}_{i=0}^{\infty}$ are functions of the normalized bandwidth parameter $c=\pi T_{c} W$. The eigenvalue $\lambda_{i}$ represents the fraction of energy of $\Phi_{i}(f)$ that is contained in the band $[-W, W]$. The $\lambda_{i}$ 's are strictly decreasing and fall off rapidly to zero with increasing $i$. For $i>2 c / \pi$, the $\lambda_{i}$ 's are essentially zero. This is illustrated in Table II, which lists eigenvalues for selected values of the parameter $c$ [19].

\section{B. Problem Reduction and Solution}

Based on the above properties of prolate spheroidal wave functions, the optimization problem formulated in (36) can be greatly simplified. First, since the $\left\{\Phi_{i}(f)\right\}_{i=0}^{\infty}$ form a complete set in $\mathcal{D}$ which is the space of functions $\Phi_{c}(f)$ of interest, we have the following expansion

$$
\Phi_{c}(f)=\sum_{m=0}^{\infty} x_{m} \Phi_{m}(f)
$$

where $x_{m}$ is given by the inner product $x_{m}=\left\langle\Phi_{c}, \Phi_{m}\right\rangle=$ $\int_{-\infty}^{\infty} \Phi_{c}(f) \Phi_{m}(f) d f$. Since we are only interested in functions $\Phi_{c}(f)$ that achieve very high energy concentration in the band $[-W, W]$, and given the fact that the eigenvalues $\lambda_{i}$ 's are rapidly decaying to zero, we can truncate the expansion of $\Phi(f)$ to a relatively short linear combination of prolate spheroidal wave functions with no major loss in accuracy. In addition, the function $\Phi_{c}(f)$ has even symmetry and therefore will only have even-indexed prolate spheroidal wave functions in its expansion. Hence, truncating to $M+1$ terms, we have

$$
\Phi_{c}(f) \simeq \sum_{m=0}^{M} x_{2 m} \Phi_{2 m}(f)
$$

where the $x_{2 m}$ 's are the coefficients of the expansion to be solved for. From Table II, it is clear that a value of $M=4$ or 5 yields sufficient accuracy for bandwidth values up to $3 / T_{c}$, which is the range of interest to us. 
Using the truncated expansion in (40) and going back to the formulation in (36), we obtain a new form for the optimization problem

$$
\begin{aligned}
\text { Minimize } & \sum_{k_{0}} \cdots \sum_{k_{M}} \alpha\left(k_{0}, \cdots, k_{M}\right) \prod_{m=0}^{M} x_{2 m}^{k_{m}} \\
\text { Subject to } & \left\{\begin{array}{l}
\sum_{m=0}^{M} x_{2 m}^{2}=T_{c} \\
\sum_{m=0}^{M} \lambda_{2 m} x_{2 m}^{2}=(1-\eta) T_{c} \\
\sum_{m=0}^{M} \beta_{2 m} x_{2 m}=0 \\
\sum_{m=0}^{M} \gamma_{2 m} x_{2 m}=0
\end{array}\right.
\end{aligned}
$$

where the sums in the objective function expression are over all combinations of the $k_{m}$ 's such that $\sum_{m=0}^{K} k_{m}=4$, and the cost coefficients $\alpha\left(k_{0}, k_{1}, \cdots, k_{M}\right)$ are given by

$$
\alpha\left(k_{0}, k_{1}, \cdots, k_{M}\right)=\left(4 ; k_{0}, k_{1}, \cdots, k_{M}\right) \int_{-\infty}^{\infty} \prod_{m=0}^{M} \Phi_{2 m}^{k_{m}}(f) d f
$$

with $\left(4 ; k_{0}, k_{1}, \cdots, k_{M}\right)$ denoting the multinomial coefficient defined as $\left(4 ; k_{0}, k_{1}, \cdots, k_{M}\right)=4 /\left(k_{0} ! k_{1} ! \cdots k_{M} !\right)$. The constraint coefficients are given by

$$
\begin{gathered}
\beta_{2 m}=\int_{-\infty}^{\infty} \Phi_{2 m}(f) \cos \left(\pi f T_{c}\right) d f \\
\gamma_{2 m}=\int_{-\infty}^{\infty} \Phi_{2 m}(f)\left(1-\cos \left(\frac{1}{2} \pi f T_{c}\right)\right) d f .
\end{gathered}
$$

We numerically solve this finite-dimensional nonlinear constrained optimization problem by means of sequential quadratic programming routines. For the generation of the prolate spheroidal wave functions, we use the results of [19].

\section{DESIGN EXAMPLES}

In this section, we give numerous design examples to illustrate the methodology to solve the optimization problem formulated above. Solutions are obtained with constraints included gradually. We first solve for $\Phi_{c}(f)$ (then $\phi_{c}(t)$ by inverse Fourier transform and subsequently $\psi_{c}(t)$ by timeshifting) while taking into account constraints 1 and 2 only (energy and bandwidth). Next, we add in the continuous-phase constraint and, finally, the quasi-uniform envelope constraint. In this way, the first solutions would achieve the best (i.e., lowest normalized mean-squared correlation $M_{c}$ ) performance since we are optimizing over a wider space of allowable functions. Then, as we add in more constraints, the lowest achievable $M_{c}$ should be increasing. However, it was found in the numerical results (to be discussed shortly) that the addition of the continuous-phase constraint did not alter results significantly. In fact, the optimization carried out with only energy and bandwidth constraints yielded near-continuous phase pulses [5] with very small phase jumps as computed from (20). Therefore, for the sake of brevity in the subsequent results, we always impose phase continuity in the optimal design, and we do not provide separate results without it.
TABLE III

Performance Measures for Optimal Chip WaVeforms Designed with Continuous-Phase AND NonUniform ENVElope

\begin{tabular}{c|c|c|c|c}
\hline $\begin{array}{c}\text { \%andwidth } \\
W T_{c}\end{array}$ & $\begin{array}{c}\text { Chip MSC } \\
M_{c}\end{array}$ & $\begin{array}{c}\text { AM Index } \\
\Delta e\end{array}$ & $\begin{array}{c}\text { SNR Gain } \\
\text { (ovcr MSK) }\end{array}$ & $\begin{array}{c}\text { Capacity } \\
\text { (vs. MSK) }\end{array}$ \\
\hline \hline \multicolumn{5}{|c}{ Results with the $99 \%$ Bandwidth Occupancy } \\
\hline \hline 1.2 & 0.2821 & $6 \%$ & $0.1 \mathrm{~dB}$ & $102 \%$ \\
\hline 1.4 & 0.2416 & $15 \%$ & $0.1 \mathrm{~dB}$ & $102 \%$ \\
\hline 1.6 & 0.2061 & $26 \%$ & $0.21 \mathrm{~dB}$ & $105 \%$ \\
\hline 1.8 & 0.1667 & $45 \%$ & $0.62 \mathrm{~dB}$ & $115 \%$ \\
\hline 2.0 & 0.1382 & $79 \%$ & $0.98 \mathrm{~dB}$ & $125 \%$ \\
\hline 2.2 & 0.1259 & $91 \%$ & $0.97 \mathrm{~dB}$ & $125 \%$ \\
\hline 2.4 & 0.1161 & $75 \%$ & $0.94 \mathrm{~dB}$ & $124 \%$ \\
\hline 2.6 & 0.1060 & $89 \%$ & $0.99 \mathrm{~dB}$ & $126 \%$ \\
\hline 2.8 & 0.0968 & $91 \%$ & $1.06 \mathrm{~dB}$ & $128 \%$ \\
\hline 3.0 & 0.0894 & $85 \%$ & $1.11 \mathrm{~dB}$ & $129 \%$ \\
\hline \hline \multicolumn{5}{|c|}{ Results with the $99.9 \%$ BaIdwidth Occupancy } \\
\hline \hline 1.6 & 0.2527 & $14.7 \%$ & $3.06 \mathrm{~dB}$ & $202 \%$ \\
\hline 1.8 & 0.2246 & $23.7 \%$ & $3.06 \mathrm{~dB}$ & $202 \%$ \\
\hline 2.0 & 0.1971 & $35.0 \%$ & $3.17 \mathrm{~dB}$ & $208 \%$ \\
\hline 2.2 & 0.1682 & $50.9 \%$ & $3.45 \mathrm{~dB}$ & $221 \%$ \\
\hline 2.4 & 0.1424 & $74.1 \%$ & $3.79 \mathrm{~dB}$ & $239 \%$ \\
\hline 2.6 & 0.1259 & $92.5 \%$ & $3.98 \mathrm{~dB}$ & $250 \%$ \\
\hline 2.8 & 0.1178 & $77.8 \%$ & $3.95 \mathrm{~dB}$ & $248 \%$ \\
\hline 3.0 & 0.1112 & $70.9 \%$ & $3.90 \mathrm{~dB}$ & $245 \%$ \\
\hline
\end{tabular}

The solutions are constructed for bandwidth occupancies starting from the minimum possible $99 \%$ and $99.9 \%$ bandwidth up to a value of $3 / T_{c}$. We reemphasize here that the zerothorder prolate spheroidal wave function $\Phi_{0}(f)$ achieves the highest fractional energy concentration in $[-W, W]$, and this fraction of energy is given by the corresponding eigenvalue $\lambda_{0}$ [16]. Therefore, the minimum possible $99 \%$ bandwidth value $W$ is the one for which $\lambda_{0}$ first reaches 0.99 . A similar statement holds for the $99.9 \%$ bandwidth as well. These values turn out to be $1.12 / T_{c}$ and $1.52 / T_{c}$ for the $99 \%$ and $99.9 \%$ bandwidth definitions, respectively.

For all subsequent results, we use MSK as a common benchmark for comparison. This is because of its relatively good performance and characteristics as discussed previously. Therefore, the SNR gains ${ }^{6}$ and capacity improvements of the optimally designed chip waveforms are all given with respect to MSK. Table III summarizes the results obtained while taking into account finite-energy limited bandwidth and continuous-phase constraints. Fig. 1 illustrates selected examples of the optimal chip waveforms obtained under these constraints. For the case of the $99 \%$ bandwidth occupancy, it is seen that modest gains in SNR of approximately $1 \mathrm{~dB}$ starting at a bandwidth $2 / T_{c}$ and up, corresponding to about $25 \%$ increase in capacity over MSK, but the penalty in envelope fluctuation is large (over 50\%). With the $99.9 \%$ bandwidth occupancy, results are better, and a gain of $3 \mathrm{~dB}$ is already achieved at about $1.6 / T_{c}$ with only about $15 \%$ envelope variation.

It is also of interest to compare the performance of the optimally designed chip waveforms among themselves by considering the minimum achievable $B M_{c}$ product as a function of the bandwidth. This is illustrated in Fig. 2 from which it is seen that performance slightly improves with increasing bandwidth and saturates at about $W=2 / T_{c}$ and $2.6 / T_{c}$ for the $99 \%$ and $99.9 \%$ bandwidth measures, respectively. Another

\footnotetext{
${ }^{6}$ We only consider the case when no thermal noise is present, i.e., $N_{0}=0$.
} 


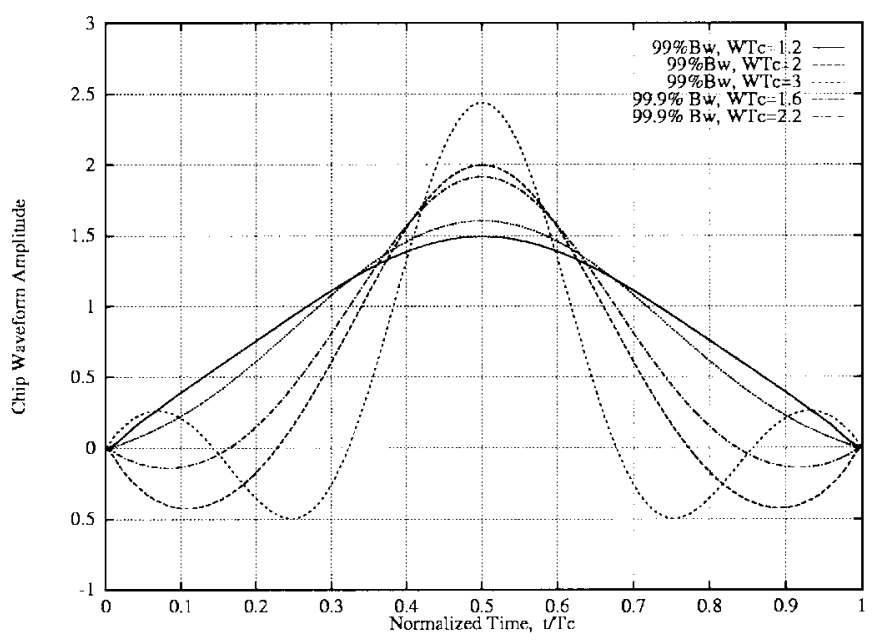

Fig. 1. Examples of optimal chip waveforms with continuous-phase nonuniform envelope for selected bandwidth values.

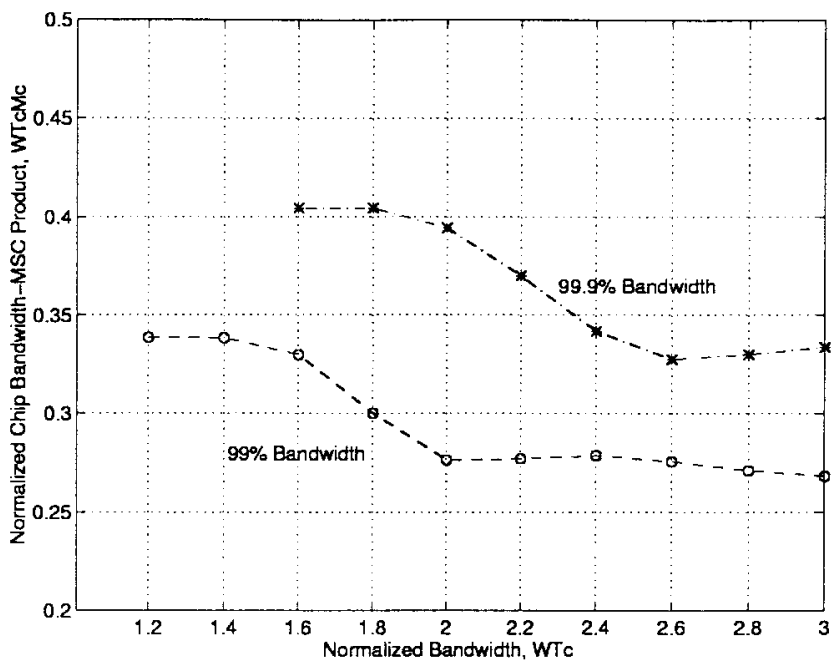

Fig. 2. Performance comparison for optimal chip waveforms with continuous-phase and nonconstant envelope.

observation to notice from Table III is the amount of envelope variation that starts to increase as more and more gain in SNR and capacity is achieved.

Table IV gives results when the optimization incorporates all constraints discussed in this paper, i.e., fixed-energy continuous-phase and quasi-constant envelope. In this case, we clearly observe a noticeable reduction in the gains achieved over MSK, especially if we use the $99 \%$ bandwidth measure. At a bandwidth $1.2 / T_{c}$, we essentially have MSK performance (the optimal pulse also resembles a half-sine). For all higher bandwidth values, performance keeps degrading. With the more stringent $99.9 \%$ bandwidth measure, the optimal pulses corresponding to low bandwidth still show some gain in SNR and capacity at the expense of modest envelope fluctuations. For example, at $1.8 / T_{c}$, there is about 2 -dB gain that correspond to $56 \%$ capacity increase and the envelope fluctuation is only $1.8 \%$. At $2.4 / T_{c}$, the envelope is virtually constant and there still is a $1-\mathrm{dB}$ SNR gain corresponding to $25 \%$ capacity improvement. For illustration, some designed chip waveforms are shown in Fig. 3 for selected values
TABLE IV

Performance Measures for Optimal Chip Waveforms Designed with Continuous-Phase AND QUASI-Uniform ENVELoPe

\begin{tabular}{|c|c|c|c|c|}
\hline $\begin{array}{c}\% \text { Bandwidth } \\
W T_{c}\end{array}$ & $\begin{array}{c}\text { Chip MSC } \\
M_{c}\end{array}$ & $\begin{array}{c}\text { AM Index } \\
\Delta \epsilon\end{array}$ & $\begin{array}{l}\text { SNR Gain } \\
\text { (over MSK) }\end{array}$ & $\begin{array}{l}\text { Capacity } \\
\text { (vs. MSK) }\end{array}$ \\
\hline \multicolumn{5}{|c|}{ Resilts with the $99 \%$ Bandwidth Occuparicy } \\
\hline 1.2 & 0.2886 & $0.2 \%$ & $0.0 \mathrm{~dB}$ & $100 \%$ \\
\hline 1.4 & 0.2632 & $0.9 \%$ & $-0.27 \mathrm{~dB}$ & $94 \%$ \\
\hline 1.6 & 0.2547 & $0.3 \%$ & $-0.71 \mathrm{~dB}$ & $85 \%$ \\
\hline 1.8 & 0.2549 & $0.4 \%$ & $-1.22 \mathrm{~dB}$ & $75 \%$ \\
\hline 2.0 & 0.2515 & $0.2 \%$ & $-1.62 \mathrm{~dB}$ & $69 \%$ \\
\hline 2.2 & 0.2427 & $0.7 \%$ & $-1.88 \mathrm{~dB}$ & $65 \%$ \\
\hline 2.4 & 0.2291 & $1.2 \%$ & $-2.01 \mathrm{~dB}$ & $63 \%$ \\
\hline 2.6 & 0.2088 & $2.5 \%$ & $-1.96 \mathrm{~dB}$ & $64 \%$ \\
\hline 2.8 & 0.1855 & $5.3 \%$ & $-1.76 \mathrm{~dB}$ & $67 \%$ \\
\hline 3.0 & 0.1605 & $10.7 \%$ & $-1.43 \mathrm{~dB}$ & $72 \%$ \\
\hline \multicolumn{5}{|c|}{ Results with the $99.9 \%$ Bandwidth Occupancy } \\
\hline 1.8 & 0.2908 & $1.8 \%$ & $1.94 \mathrm{~dB}$ & $156 \%$ \\
\hline 2.0 & 0.2861 & $3.5 \%$ & $1.55 \mathrm{~dB}$ & $143 \%$ \\
\hline 2.2 & 0.2864 & $0.4 \%$ & $1.14 \mathrm{~dB}$ & $130 \%$ \\
\hline 2.4 & 0.2720 & $0.3 \%$ & $0.98 \mathrm{~dB}$ & $125 \%$ \\
\hline 2.6 & 0.2606 & $0.1 \%$ & $0.82 \mathrm{~dB}$ & $121 \%$ \\
\hline 2.8 & 0.2487 & $0.7 \%$ & $0.70 \mathrm{~dB}$ & $118 \%$ \\
\hline 3.0 & 0.2361 & $2.0 \%$ & $0.63 \mathrm{~dB}$ & $116 \%$ \\
\hline
\end{tabular}

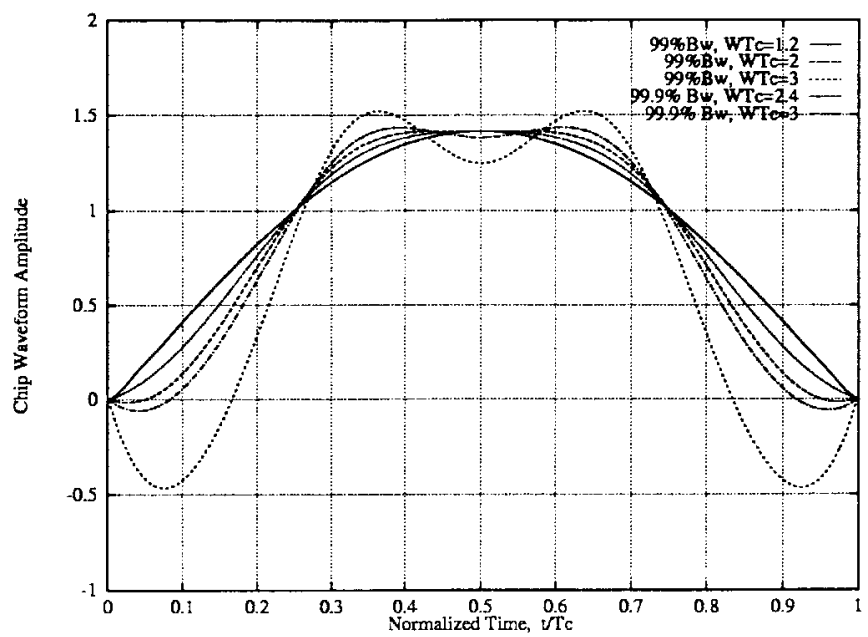

Fig. 3. Examples of optimal chip waveforms with continuous-phase and quasi-uniform envelope for selected bandwidth values.

of bandwidth occupancies. The observation that optimal waveforms for this case perform well only at lower bandwidth values is further illustrated in Fig. 4, which is clearly different than in the case of 2 .

Finally, we present plots for benchmarking the conventional modulations that we introduced in Section III (MSK, SFSK, and TDRC) with the optimally designed chip waveforms. In Figs. 5 and 6, we show results for the first optimization case (i.e., with phase continuity but without envelope uniformity). It is again seen from these plots that MSK and TDRC are quasi-optimal with regard to the $99 \%$ bandwidth measure, while with the $99.9 \%$ one, only TDRC retains its quasioptimality. However, in the case of envelope near-uniformity (see Figs. 7 and 8), SFSK performs consistently better with both the $99 \%$ and $99.9 \%$ bandwidth measures. We finally note, in this last case, that the observation that TDRC seems to "beat" the optimal limiting curves is in fact overlooking the $17 \%$ envelope variation of this modulation format. 


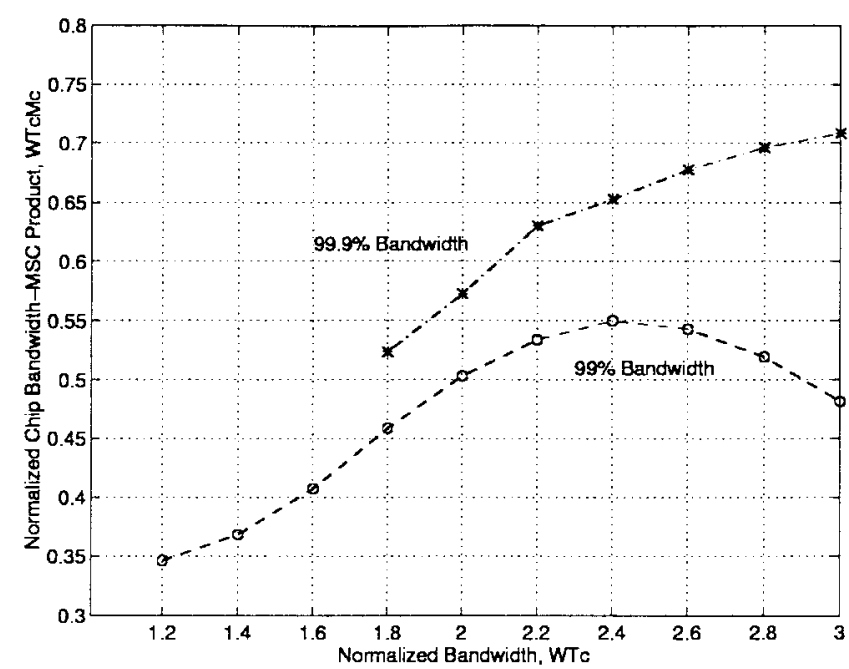

Fig. 4. Performance comparison of optimal chip waveforms with continuous-phase and quasi-uniform envelope.

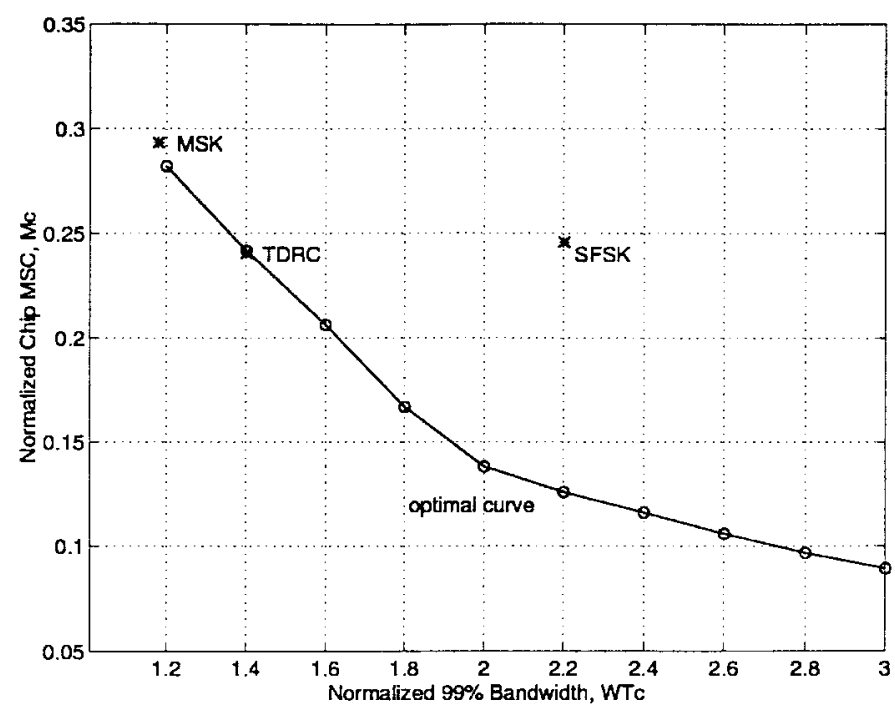

Fig. 5. Optimal performance limit ( $99 \%$ bandwidth measure) with continuous-phase and nonuniform envelope.

\section{CONCLUSION}

This paper outlined a comprehensive methodology to design DS-CDMA chip waveform shapes that achieve minimal multiple-access interference power (or equivalently, normalized chip mean-squared correlation), while at the same time satisfying various desirable constraints on the transmitted signals, including limited-power bandwidth occupancy, continuous-phase and low envelope fluctuation. The techniques presented were based on a formulation using special basis functions known as prolate spheroidal wave functions, which reduced the problem to an equivalent discrete finitedimensional nonlinear optimization that was easy to solve by standard numerical techniques.

Several design examples were given based on this methodology. In particular, sets of optimal waveforms were constructed for increasing values of the $99 \%$ and $99.9 \%$ bandwidth measures. It was found that the minimum-achievable chip meansquared correlation decreased as the allocated inband power

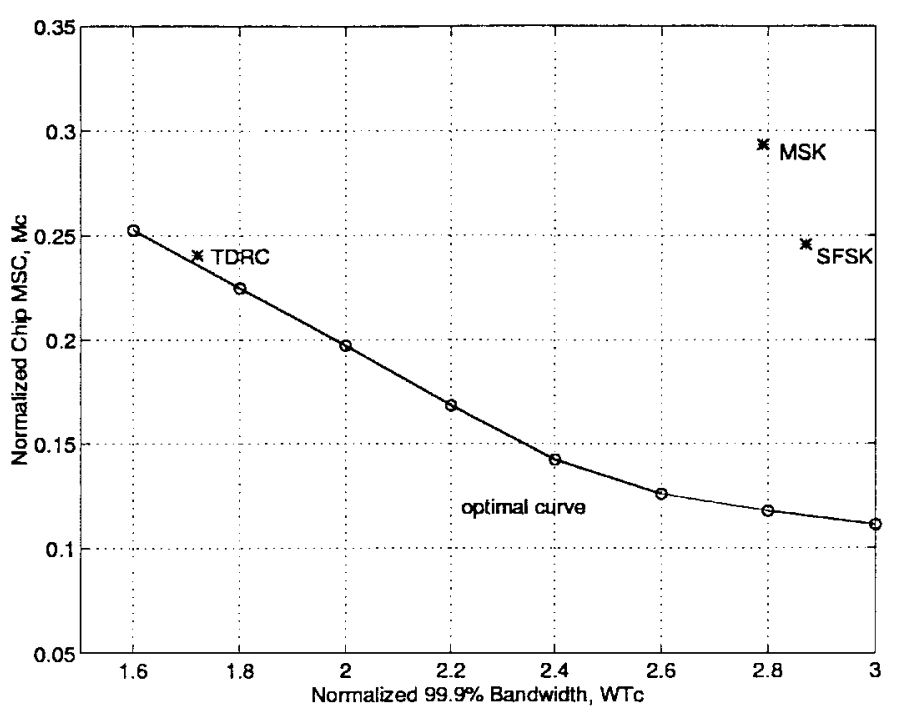

Fig. 6. Optimal performance limit (99.9\% bandwidth measure) with continuous-phase and nonuniform envelope.

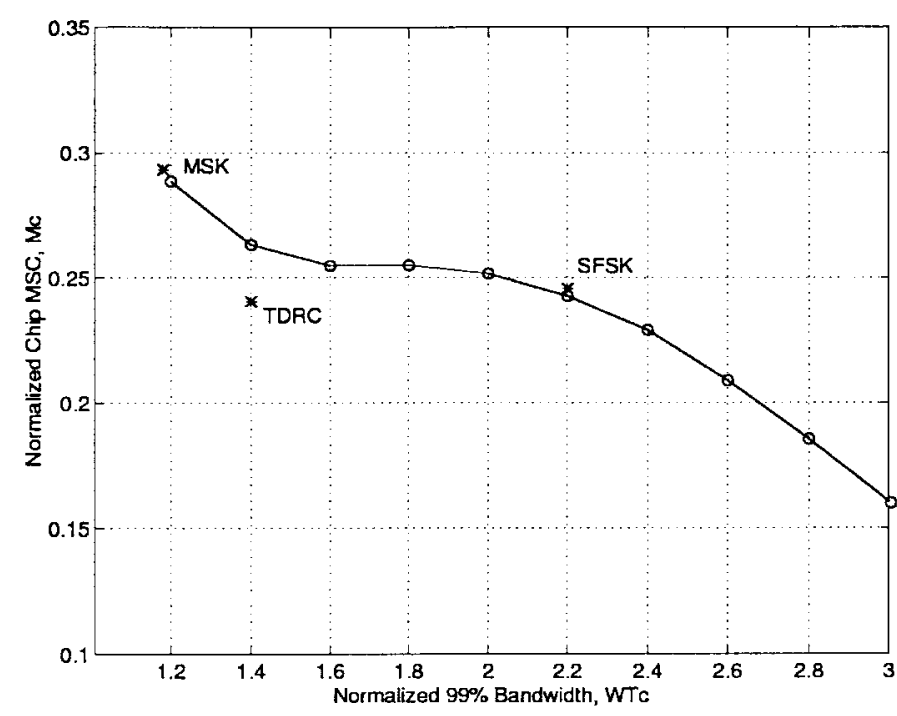

Fig. 7. Optimal performance limit ( $99 \%$ bandwidth measure) with continuous-phase and quasi-uniform envelope.

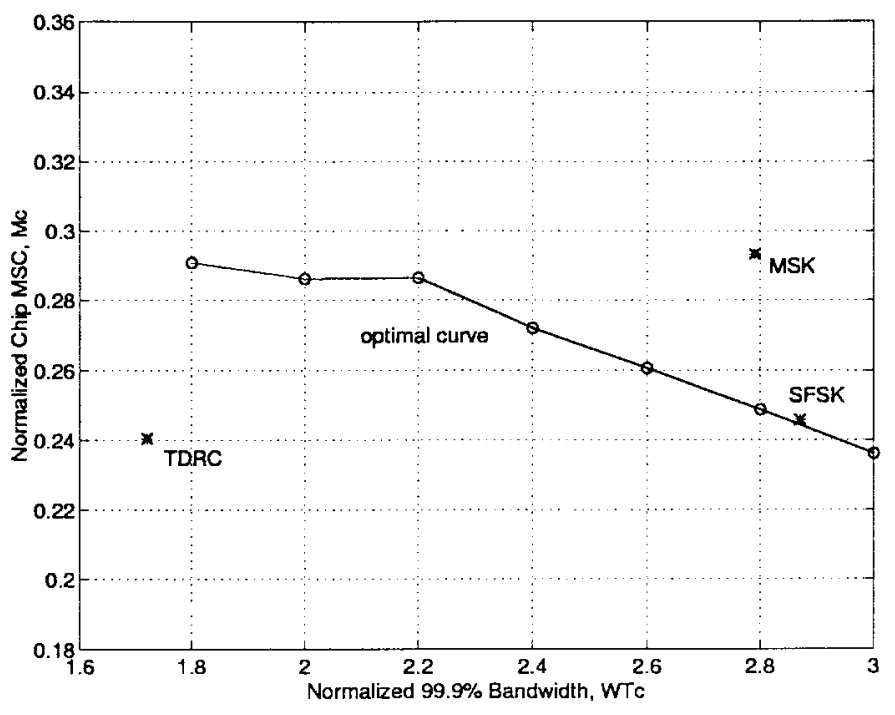

Fig. 8. Optimal performance limit (99.9\% bandwidth measure) with continuous-phase and quasi-uniform envelope. 
bandwidth increased. In addition, the impact of added phase continuity was found to be minimal. However, the requirement on low envelope fluctuation caused considerable increase in the lowest achievable chip mean squared correlation. Interesting design tradeoffs arise from these conflicting trends of SNR (or capacity) improvement versus envelope fluctuation. For example, if highly linear power amplifiers are available, then one need not worry about envelope uniformity since these amplifiers will not cause spectral sidelobe regeneration. In practice, such amplifiers can be used at the base station where power efficiency is not a major issue, and substantial gains in performance are then achieved by using the optimally designed chip waveforms. However, for the battery-powered mobile handset, power efficiency is crucial and nonlinear amplifiers are usually preferred, in which case envelope uniformity becomes an issue. It may not be advantageous to use a pulse which achieves high capacity and at the same time suffers great envelope nonuniformity. It should be noted, however, that in this case, definite conclusions cannot be made before studying the additional effects of band-limiting filters, which we are considering in a future study.

Finally, we can say that for all practical purposes, MSK is quasi-optimal with regard to the $99 \%$ bandwidth measure, while the raised-cosine pulse is equally good with both the 99\% and $99.9 \%$ measures, but at the expense of some envelope variation. On the other hand, SFSK is quasi-optimal, with regard to the $99.9 \%$ bandwidth occupancy, among the class of constant-to-low envelope variation pulses.

\section{REFERENCES}

[1] M. B. Pursley, "Spread-Spectrum Multiple-Access Communications," in Multi-User Communications (CISM Courses and Lectures no. 265), G. Longo, Ed. New York: Springler-Verlag, 1981.

[2] M. B. Pursley, F. D. Garber, and J. S. Lehnert, "Analysis of generalized quadriphase spread-spectrum communications," in Proc. IEEE Int. Conf. Communications, June 1980, vol. 1, pp. 15.3.1-15.3.6.

[3] J. S. Lehnert, "Chip waveform selection in offset-quaternary directsequence spread-spectrum multiple-access communications," M.S. thesis, Univ. of Illinois, Urbana, IL, 1981

[4] M. A. Landolsi and W. E. Stark, "DS/CDMA chip waveform design for optimal power-bandwidth performance," in Proc. 6th Int. Symp. Personal, Indoor and Mobile Radio Communications, Toronto, ON, Sept. 1995, pp. 706-710.

[5] _ "Optimal chip waveforms for DS/CDMA with generalized offset quadrature phase shift keying," Eur. Trans. Telecommun., vol. 8, no. 5, Sept./Oct. 1997

[6] S. Pasupathy, "Minimum shift keying: A spectrally efficient modulation," IEEE Commun. Mag., vol. 17, pp. 14-22, July 1979.

[7] F. Amoroso, "Pulse and spectrum manipulation in the minimum (frequency) shift keying (MSK) format," IEEE Trans. Commun., vol. COM-24, pp. 381-384, Mar. 1976.

[8] R. Anjaria and R. Wyrwas, "The effect of chip waveform of CDMA systems in multipath fading noisy channels," in Proc. IEEE 42nd VTS Conf., Denver, CO, May 1992, pp. 672-675.

[9] A. J. Vigil, M. A. Belkerdid, and D. C. Malocha, "Application of classical cosine series window functions to full-response signaling offset quadrature modulation systems," IEEE Trans. Commun., vol. 41, pp. 11-15, Jan. 1993.

[10] H. Miyakawa and H. Imai, "On Pulse Waveforms for Asynchronous CDM Communications," in Proc. 1068 Joint Convention Rec. Inst. of Elect. Eng., Japan, Oct. 1968, p. 2333.
[11] A. H. Nuttall, "Minimum rms bandwidth of $M$ time-limited signals with specified code or correlation matrix," IEEE Trans. Inform. Theory, pp. 699-707, Sept. 1968

[12] E. S. Sousa and V. M. DaSilva, "Multicarrier orthogonal CDMA signals for quasisynchronous communication systems," IEEE J. Select. Areas Commun., vol. 12, pp. 842-852, June 1994

[13] P. I. Dallas and F. N. Pavlidou, "Innovative chip waveforms in microcellular DS/CDMA packet mobile radio," IEEE Trans. Commun., vol. 44, pp. 1413-1416, Nov. 1996.

[14] F. Amoroso, "Investigation of signal variance, bit error rates, and pulse dispersion for DSPN signaling in a mobile dense scatterer ray tracing model,” Int. J. Satellite Commun., vol. 12, no. 6, pp. 579-588, Nov./Dec. 1994.

[15] S. Ariyavisitakul and T.-P. Liu, "Characterizing the effects of nonlinear amplifiers on linear modulation for digital portable radio communications," IEEE Trans. Veh. Technol., vol. 39, pp. 383-389, Nov. 1990.

[16] D. Slepian and H. O. Pollak, "Prolate spheroidal wave functions, Fourier analysis and uncertainty-I," Bell Syst. Tech. J., vol. 40, no. 1, pp. 43-64, Jan. 1961.

[17] H. J. Landau and H. O. Pollak, "Prolate spheroidal wave functions, Fourier analysis and uncertainty-II," Bell Syst. Tech. J., vol. 40, no. 1, pp. 65-84, Jan. 1961.

[18] A. J. Viterbi, CDMA: Principles of Spread Spectrum Communication Reading, MA: Addison-Wesley, 1995.

[19] C. Flammer, Spheroidal Wave Functions. Stanford, CA: Stanford Univ Press, 1957.

[20] J. B. Anderson, T. Aulin, and C.-E. Sundberg, Digital Phase Modulation. New York: Plenum, 1986.

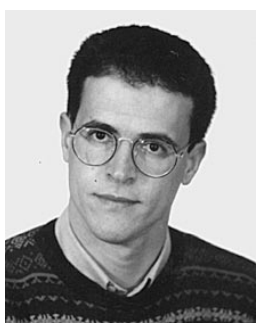

Mohamed A. Landolsi received the Diplôme d'Ingénieur degree from L'Ecole Nationale d'Ingénieurs de Tunis, Tunisia, in 1988, and the M.S.E. and Ph.D. degrees in electrical engineering from the University of Michigan, Ann Arbor, MI, in 1991 and 1996, respectively.

Since February of 1995, he has been with Nortel Networks (formerly Northern Telecom) as a Member of Scientific Staff and has held research positions within different groups including Wireless Systems Engineering, Wireless Advanced Technology, and Broadband Satellite Access. His current research interests are in the areas of system design and performance analysis of land mobile cellular systems and VSAT broadband satellite networks employing CDMA and TDMA technologies.

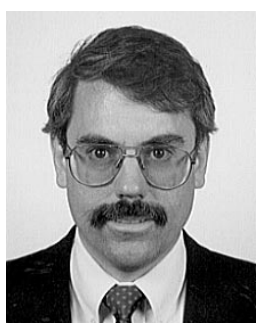

Wayne E. Stark (S'77-M'82-SM'94-F'98) received the B.S. (with highest honors), M.S., and $\mathrm{Ph} . \mathrm{D}$. degrees in electrical engineering from the University of Illinois, Urbana, in 1978, 1979, and 1982, respectively.

Since September 1982, he has been a faculty member in the Department of Electrical Engineering and Computer Science at the University of Michigan, Ann Arbor, MI. He was involved in the planning and organization of the 1986 International Symposium on Information Theory which was held in Ann Arbor, MI. His research interests are in the areas of coding and communication theory, especially for spread-spectrum and wireless communication networks.

Dr. Stark is a member of Eta Kappa Nu, Phi Kappa Phi and Tau Beta Pi. He was selected by the National Science Foundation as a 1985 Presidential Young Investigator. From 1984 to 1989, he was the Editor for Communication Theory of the IEEE TRANSACtions on Communications in the area of Spread-Spectrum Communications. 